\title{
INFRARED EXTRAGALACTIC BACKGROUND LIGHT
}

\author{
Toshio Matsumoto \\ Department of Astrophysics \\ Nagoya University \\ Chikusa-ku, Nagoya, Japan 464-01
}

\begin{abstract}
Infrared extragalactic background light plays an important role in the study of the early history of the universe, especially as a probe to search for the primeval galaxies. In the near-infrared region, UV and visible light emitted from high redshift galaxies could be observable. Measurement of the sky fluctuation at $2.2 \mu \mathrm{m}$ gives a very low upper limit. The rocket observation of the near-infrared diffuse emission reveals isotropic emission which is possibly ascribed to an extragalactic origin. The observed brightness and fluctuation are not consistent with the standard scenario of the primeval galaxies. In the far-infrared region, integrated light of dust emission of the distant galaxies forms another cosmic background radiation. IRAS and the Nagoya-Berkeley rocket experiment found a clear correlation between HI column density and far-infrared sky brightness; however, there remains an uncorrelated isotropic emission component. If we ascribe this emission to extragalactic origin, a fairly big evolution effect is required. In the submillimeter region, excess emission over the $2.74 \mathrm{~K}$ blackbody spectrum was found, which requires the vast energy generation in the early universe.
\end{abstract}

\section{INTRODUCTION}

The light of the night sky has been one of the important astronomical objects, concerning Olbers's paradox. Partridge and Peebles (1967) first proposed a theoretical formulation taking the formation and evolution of the galaxies into account. Since then, many people have tried to detect the extragalactic background light (EBL); however, observations were limited in the optical region. The recent development of space experiments has made it possible to observe the infrared EBL and has revealed its importance to cosmology.

The infrared EBL could provide an additional and a different kind of information for cosmology. Compared with the optical region, the near-infrared region is advantageous because of less bright foreground emission. Redshifted UV and optical radiation from the primeval galaxies could be observed in the near-infrared region $(1-5 \mu \mathrm{m})$, if the galaxy formation occurred at a high redshift era. In the mid-infrared region $(10-50 \mu \mathrm{m})$ thermal emission of the interplanetary dust is too strong to observe the EBL, but foreground emission becomes very dark at $\lambda>100 \mu \mathrm{m}$. The far-infrared and submillimeter regions provide an opportunity to observe the different kinds of EBL, which originated in the dust emission of the distant galaxies. The far-infrared EBL has a complementary nature to the near-infrared EBL, and both will render observational evidence on galaxy formation.

In this paper we review the present status of the observation of the infrared EBL. In section 2, the foreground emission is summarized. Sections 3 and 4 present the observational results on the near-infrared and the far-infrared EBL, respectively. Future prospects of the infrared EBL are discussed in section 4. 


\section{FOREGROUND EMISSION}

The EBL is supposed to be isotropic over the whole sky. In order to obtain the EBL from the observed sky brightness, foreground diffuse emission must be carefully subtracted. In the infrared region terrestrial emission causes serious problems. The airglow emission of atmospheric molecules $\left(\mathrm{OH}, \mathrm{CO}, \mathrm{CO}_{2}\right.$, etc.) is prominent in the near-infrared region, while thermal emission of the atmosphere is conspicuous in the far-infrared region. These terrestrial emissions make it extremely difficult to observe the infrared EBL from the ground. Space observations with cooled optics are absolutely essential.

Figure 1 indicates the estimated sky brightness of several extraterrestrial emission components for the darkest region of the sky.

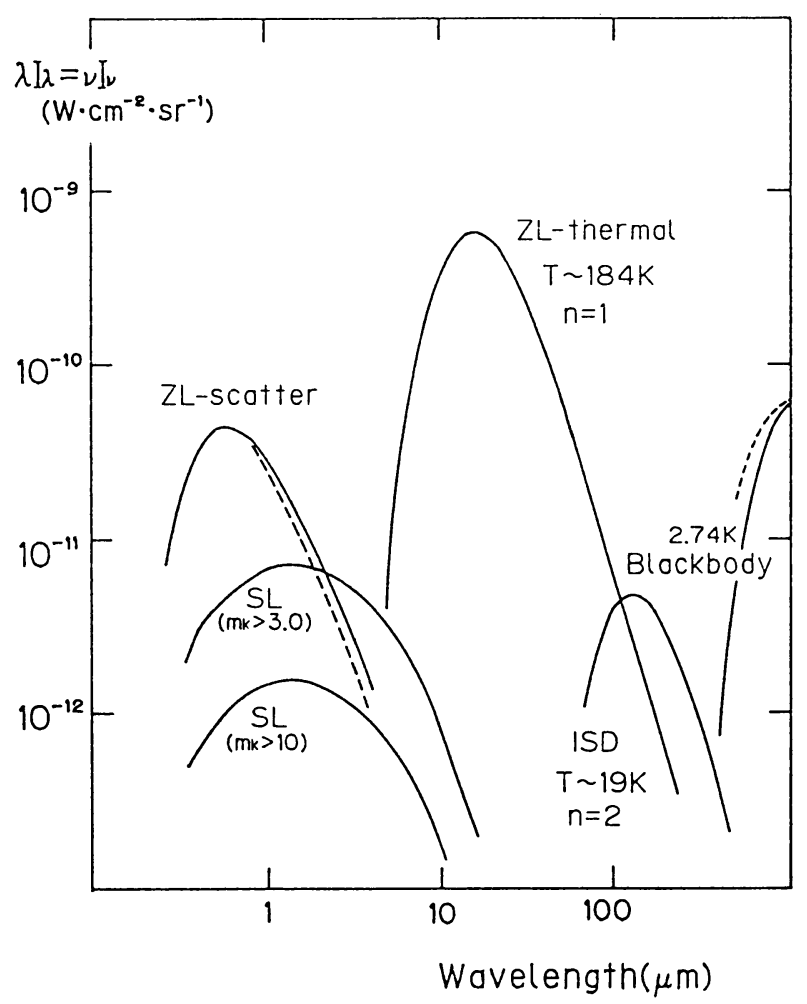

Figure 1. The estimated brightness of the extraterrestrial foreground emission. SL (star light) means the integrated light of the faint stars in the Galaxy. ZL-scatter and ZL-thermal indicate the scattered sunlight by the interplanetary dust (IPD) and the thermal emission of the IPD, respectively. ISD implies the thermal emission of the interstellar dust which correlates with the column density of neutral hydrogen. The $2.74^{\circ} \mathrm{K}$ blackbody spectrum and the recently discovered submillimeter excess (dashed line) are also indicated.

Interplanetary dust causes two kinds of diffuse emission. One is the zodiacal light; that is, scattered sunlight. ZL-scatter in Figure 1 is obtained by extrapolating the optical data at the ecliptic pole assuming the solar color (Hoffman et al.; 1973, Hayakawa et al., 1970). Based on 
recent near-infrared rocket observation, Akiba et al. (1989) reported that the infrared color of the zodiacal light is bluer than the solar color at the ecliptic pole region, as is shown by the dashed line in Figure 1. The near-infrared feature of the zodiacal light is not so well understood yet and more extensive studies are required.

Another interplanetary emission component is thermal emission of the interplanetary dust (ZL-thermal in Figure 1). IRAS extensively observed the ZL-thermal and the result at the ecliptic pole is shown in Figure 1, adopting a dust temperature of $184^{\circ} \mathrm{K}$ and an emissivity index of 1.0 (Hauser et al. 1984). Since IRAS observed only the sky at the solar elongation angle of 90 degrees, the spatial distribution is not well understood yet. Nevertheless, it should be mentioned that the ZL-scatter is so bright that the EBL at the wavelength region of $10-$ $100 \mu \mathrm{m}$ is heavily masked. This makes it extremely difficult to detect the far-infrared EBL.

Integrated light of the faint stars, star light (SL), forms a diffuse extended emission. Figure 1 shows the estimated brightness of SL at the galactic pole region. The spectrum of the SL is taken from the color of $S_{b c}$ type galaxies (Yoshii and Takahara 1988) and the absolute value by Matsumoto et al. (1988b). Brightness of the SL strongly depends on the magnitude of the faintest star which can be identified as a point source. Two cases, corresponding to the limiting magnitude $m_{K}=3.0 \mathrm{mag}$ and $10.0 \mathrm{mag}$, are indicated in Figure 1. In order to reduce the SL component, a large aperture with a small beam is desirable. At high galactic latitude, spatial distribution of SL is essentially represented by $\operatorname{cosec}(b)$ law (Matsumoto et al. 1988b).

Interstellar dust (ISD) heated by the interstellar radiation field also forms far-infrared and submillimeter diffuse emission. IRAS and the Nagoya-Berkeley rocket experiment have revealed that the ISD emission is fairly well correlated with the column density of neutral hydrogen, $\mathrm{N}(\mathrm{HI})$. In Figure 1, ISD emission is estimated assuming $\mathrm{N}(\mathrm{HI})$ of $1.5 \times 10^{20} \mathrm{~cm}^{-2}$ and a dust temperature of $19^{\circ} \mathrm{K}$ (Lange et al., 1989).

At the right end of Figure 1 , the spectrum of the $2.74^{\circ} \mathrm{K}$ blackbody is also shown for comparison. The dashed line over the $2.74^{\circ} \mathrm{K}$ blackbody spectrum indicates the result of the Nagoya-Berkeley rocket experiment (Matsumoto et al., 1988a).

Figure 1 clearly shows that there are two windows through the Galaxy to observe EBL. One is the near-infrared and the other is the submillimeter region.

\section{NEAR-INFRARED OBSERVATIONS}

At wavelengths shorter than the $\mathrm{K}$ band, observations of EBL from ground-based telescopes have been carried out. In this region atmospheric emission is rather weak but airglow is still prominent. Boughn and Kuhn (1986) have tried to detect the EBL using the eclipse by dark clouds. They found that the dark cloud was brighter than the nearby reference sky and its level was $4 \times 10^{-12} \mathrm{~W} \mathrm{~cm}^{-2} \mathrm{sr}^{-1}$. This is due to the reflected galactic light, and the contribution of the EBL is not certain. Boughn et al. (1986) observed the smoothness of the sky at $2.2 \mu \mathrm{m}$ and obtained the upper limit of $10^{-12} \mathrm{~W} \mathrm{~cm}^{-1} \mathrm{sr}^{-1}$ for a $10-30^{\prime \prime}$ beam and $4 \times 10^{-12} \mathrm{~W} \mathrm{~cm}^{-2} \mathrm{sr}^{-1}$ for a $60-300^{\prime \prime}$ beam. Assuming a model for primeval galaxies they excluded the shaded area of Figure 2, in which the K-magnitude and the number density of primeval galaxies are plotted. Obtained upper limits constrain the model but are still marginally consistent with it.

As described before, space observation with cooled optics is very advantageous in observing the near-infrared EBL. Harwit et al. (1966) carried out rocket observations; however, the detector sensitivity was not good enough, resulting in a rather high upper limit for the EBL. Hoffman and Lemke (1978) analyzed the data obtained from their balloon-borne telescope and obtained an upper limit of $6 \times 10^{-10} \mathrm{~W} \mathrm{~cm}^{-2} \mathrm{sr}^{-1}$ at $2.4 \mu \mathrm{m}$. This implies that 


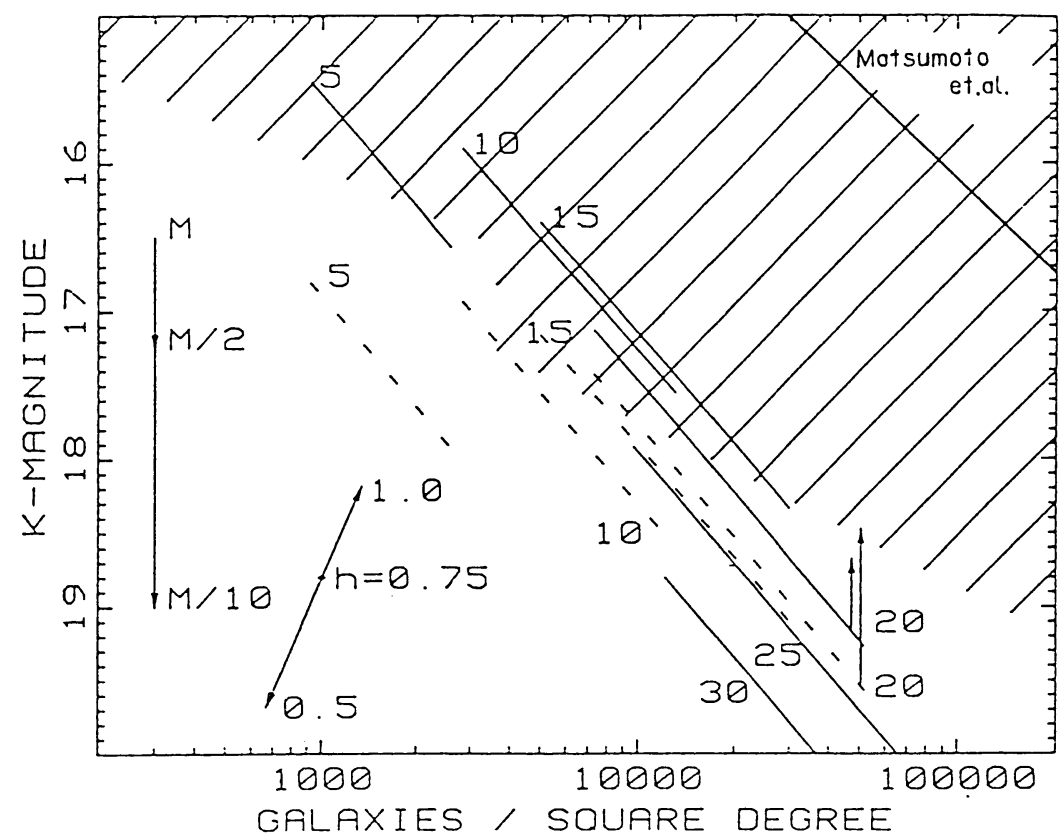

Figure 2. Models of primeval galaxies excluded by the measurement of the smoothness of the sky at $2.2 \mu \mathrm{m}$ (shaded area). The solid line labeled Matsumoto et al. is their measured brightness.

balloon altitudes are not high enough to make an absolute measurement of the near-infrared EBL.

Matsumoto et al. (1988b) have recently carried out a rocket experiment to search for the near-infrared EBL with a liquid-nitrogen-cooled telescope. Figure 3 shows the observed sky brightness at the galactic north pole region. Open circles represent the results of the standard astronomical filter bands, $\mathrm{H}, \mathrm{J}, \mathrm{K}, \mathrm{L}$, and $\mathrm{M}$, whose data were taken throughout the flight. Crosses represent the data of the filter bands on the rotating wheel, which were changed intermittently during the flight. This caused slightly larger error bars for the crosses than those for the open circles. As is shown in Figure 3, the $\mathrm{J}$ band data can be well explained as the sum of the SL and the ZL-scatter. The spatial variation of the observed sky brightness indicates that the SL essentially obeys a $\operatorname{cosec}(b)$ law, which is consistent with the model of the Galaxy. At the $\mathrm{K}$ band, the observed brightness is considerably brighter than that extrapolated from the $\mathrm{J}$ band. The observed spatial variation indicates that the SL is consistent with the model and the ZL-scatter has a little bluer color than the solar color. As a result there remains an isotropic emission component in the $\mathrm{K}$ band. Similar isotropic emission components are found in the wavelength region of $3-4 \mu \mathrm{m}$.

Figure 4 indicates the isotropic emission thus obtained. There are several significant data points; however, a careful study is required to conclude the EBL origin for the observed isotropic emission. One serious issue is the environmental emission surrounding the rocket body. Before apogee, some emission lines were observed which had a decay time constant of $41 \mathrm{~s}$. The environmental emission was prominent at wavelengths longer than $3 \mu \mathrm{m}$, especially the $3.5 \mu \mathrm{m}$ band due to the $\mathrm{CH}$-bond of the organic materials was clearly seen. The contribution of the environmental emission was estimated assuming exponential decay, which resulted in a 


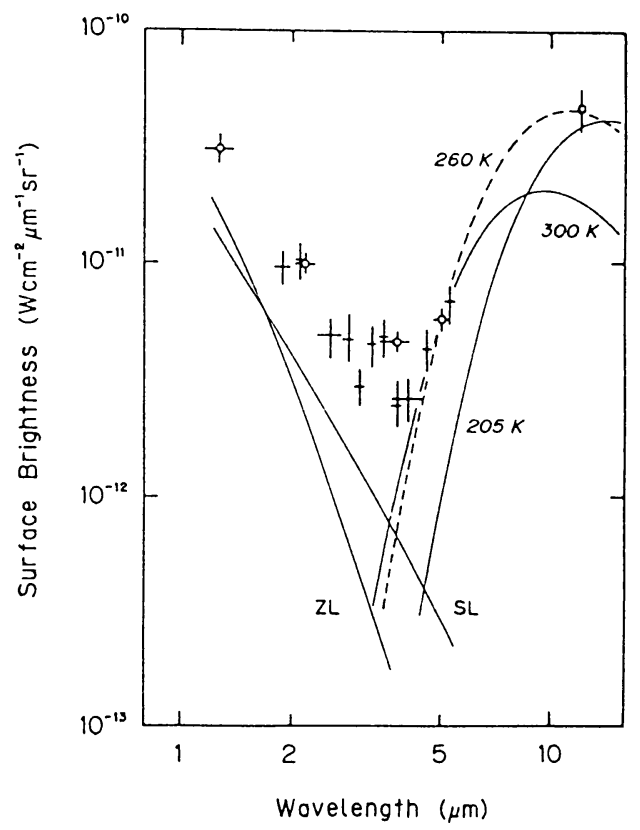

Figure 3. Near-infrared sky brightness at the galactic north pole region.

negligible effect after apogee. Nevertheless, environmental emissions can not be excluded completely as an origin for the isotropic emission at wavelengths longer than $3 \mu \mathrm{m}$. On the other hand, the isotropic emission around the $\mathrm{K}$ band is almost free from environmental emission and there is no bright atomic and molecular line in this band. As for the $\mathrm{K}$ band, a small possibility is left that the ZL-scatter has an isotropic offset brightness only in the $\mathrm{K}$ band, although this is not consistent with previous measurements of the interplanetary dust and cometary dust. Taking these ambiguities into account, Matsumoto et al. (1988b) concluded that the isotropic emission at the $\mathrm{K}$ band can be attributed to an extragalactic origin, but other isotropic emission could be a marginal detection of the EBL.

In the case that the isotropic emission observed by Matsumoto et al. (1988b) is extragalactic in origin, its effect on cosmology is enormous. In Figure 4, models of the EBL for two extreme cases by Partridge and Peebles (1967) are shown. Model 4 assumes that all helium was synthesized during the galaxy formation era, while model 1 assumes no evolution for galaxies. The isotropic emission in Figure 4 is close to that of model 4, which indicates the vast energy generation in the early universe. There seems to be a line feature at the $\mathrm{K}$ band showing an anomalous spectral feature. Matsumoto et al. (1988b) suggested its origin to be a redshifted Lyman $\alpha$ or Lyman-limit.

The isotropic emission at the $\mathbf{K}$ band should be compared with the smoothness of the sky. In Figure 2, a solid line labeled as Matsumoto et al. shows the allowable position which gives the observed brightness for isotropic emission. The observed brightness is so bright that it lies in the region (shaded area) excluded by the model. Further, the observed isotropic emission should be consistent with the fluctuation of the $2.2 \mu \mathrm{m}$ sky brightness, which requires the number density of the primeval galaxies to be $\geq 100$ galaxies in a 10 " beam. In this case, the apparent $\mathrm{K}$ magnitude of the primeval galaxies should be larger than $21.3 \mathrm{mag}$. The 


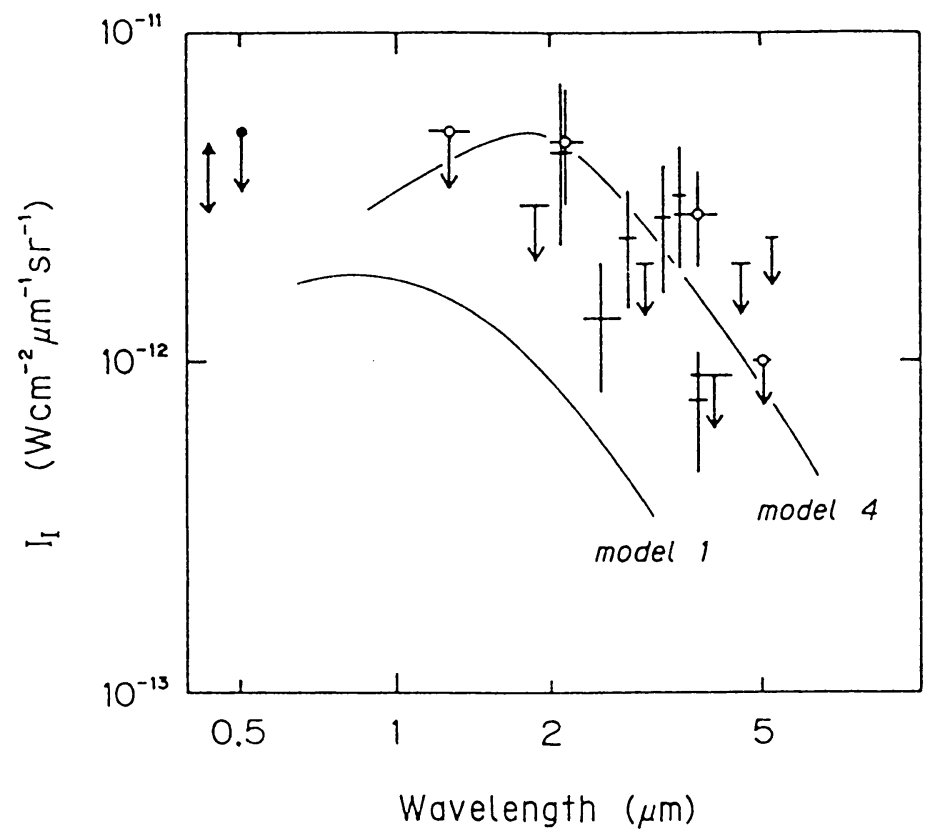

Figure 4. The near infrared isotropic emission observed by Matsumoto et al. (1988b). Upper limits in the optical region by Dube et al. (1977): filled circle; and Toller (1983): triangle are also shown. The EBL calculated by Partridge and Peebles (1967) for two extreme cases is indicated by solid lines.

feature of the primeval galaxies thus obtained is far from that in Figure 2 and could hardly be explained by the standard scenario on the formation and evolution of the galaxies.

\section{FAR-INFRARED AND SUBMILLIMETER OBSERVATIONS}

At wavelengths longer than $100 \mu \mathrm{m}$ a possibility to detect the EBL appears again. IRAS has attained a whole sky survey and found a clear correlation between the diffuse infrared emission and the column density of neutral hydrogen, $\mathrm{N}(\mathrm{HI})$, in all wavelength bands. In these correlation diagrams, linear fits do not cross the origin and there remains a considerable amount of isotropic emission which does not correlate with $\mathrm{N}(\mathrm{HI})$. In the three short wavelength bands, this isotropic emission could be ascribed to the ZL-thermal; however, isotropic emission at the $100 \mu \mathrm{m}$ can hardly be explained by known astronomical emission components (Boulanger and Perault, 1988).

Although IRAS was not well designed to observe the diffuse isotropic emission, a similar isotropic emission was found in the Nagoya-Berkeley rocket experiment (Matsumoto et al., 1988a; Lange et al., 1989). Figure 5 shows the correlation diagram between $N(H I)$ and the infrared brightness for three short wavelength channels. In this experiment, a circle on the sky centered at $l=203.2 \pm 1.5^{\circ}, b=34.9 \pm 1.5^{\circ}$ of a radius of $15.7 \pm 1^{\circ}$, was scanned with a $7.6^{\circ}$ beam. Figure 5 shows a tight correlation, the same as observed by IRAS. The gradients of the fitted straight lines provide ratios of the infrared brightness to $\mathrm{N}(\mathrm{HI})$, which are summarized and compared with the IRAS result in Table 1 . The result at $102 \mu \mathrm{m}$ is consistent with that by Terebey and Fich (1986) but a little lower than that by Boulanger and Perault (1988). 
If we assume that the infrared brightness correlated with $\mathrm{N}(\mathrm{HI})$ is due to the thermal emission of the interstellar dust, the observed spectrum can be fitted with a temperature of $17 \pm 3^{\circ} \mathrm{K}$ and a spectral index of 2 .

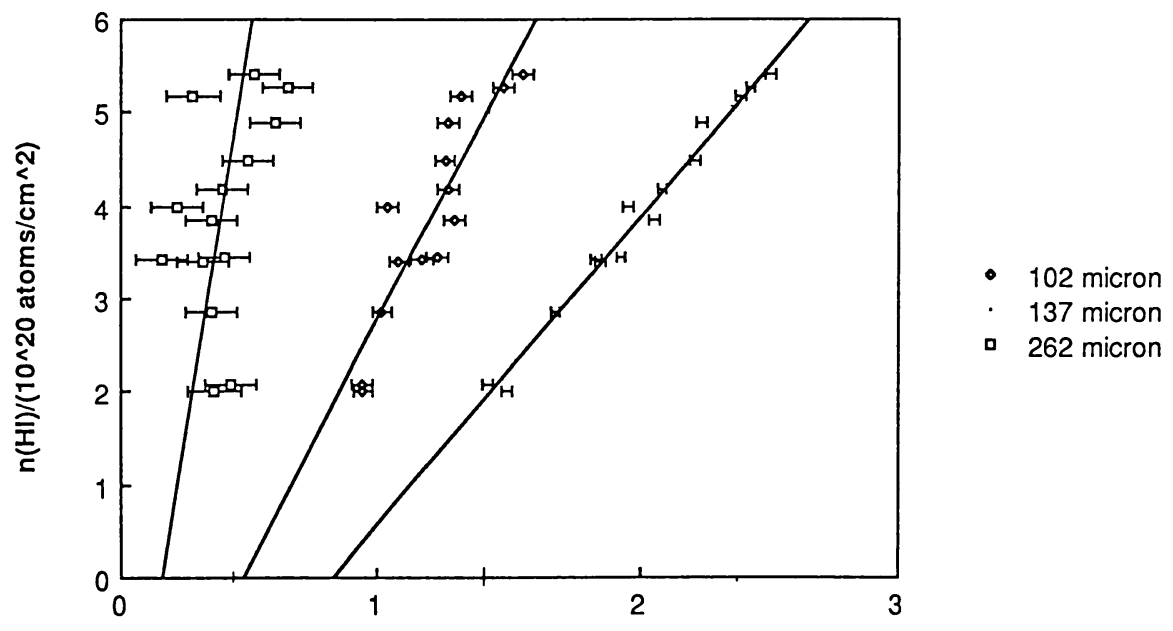

IR Brightness/(10^-11 W/cm^2 sr)

Figure 5. Correlation between the infrared sky brightness and the column density of neutral hydrogen, N(HI).

TABLE 1. Ratios of the infrared sky brightness to the column density of the neutral hydrogen, $\mathrm{N}(\mathrm{HI})$, in units of (MJy) $\mathrm{cm}^{-2} \mathrm{sr}^{-1} / 10^{22} \mathrm{H}$.

\begin{tabular}{ccc}
\hline \hline Nagoya-Berkeley & & \\
\hline Ch. $6(102 \mu \mathrm{m})$ & Ch. $5(137 \mu \mathrm{m})$ & Ch. $4(262 \mu \mathrm{m})$ \\
$0.65 \pm 0.14$ & $1.4 \pm 0.3$ & $0.5 \pm 0.25$ \\
\hline IRAS $(100 \mu \mathrm{m})$ & & \\
\hline $0.64 \pm 0.11$ & Terebey and Fich & $1 \sim 125^{\circ},|\mathrm{b}|<10^{\circ}$ \\
$0.42 \pm 0.08$ & Terebey and Fich & $1 \sim 215^{\circ},|\mathrm{b}|<10^{\circ}$ \\
$1.4 \pm 0.2$ & Boulanger and Perault & $27.5^{\circ}<\mathrm{b}<32.5^{\circ}$ \\
$1.1 \pm 0.1$ & Boulanger and Perault & $-32.5^{\circ}<\mathrm{b}<-27.5^{\circ}$ \\
$0.92 \pm 0.14$ & Boulanger and Perault & $\mathrm{b}>50^{\circ}$ \\
$0.79 \pm 0.06$ & Boulanger and Perault & $\mathrm{b}<-50^{\circ}$ \\
\hline
\end{tabular}

Figure 5 indicates that there remains an offset brightness even if $\mathrm{N}(\mathrm{HI})=0$. Concerning this residual isotropic emission, the scatter of the data points in Figure 5 provides valuable information. At $102 \mu \mathrm{m}$, the scatter from the fitted straight line is larger than that at $137 \mu \mathrm{m}$, but correlated to it. This suggests that the isotropic emission at $102 \mu \mathrm{m}$ is due to the thermal emission of the interplanetary dust, that is, ZL-thermal. Since the observed sky was anti-solar region, direct comparison with IRAS is not possible; however, it seems plausible to attribute 
the whole of the residual emission at $102 \mu \mathrm{m}$ to interplanetary origin. At $137 \mu \mathrm{m}$, the situation is not so simple, since even the graybody spectrum for the ZL-thermal cannot explain the residual isotropic emission. There remains an unknown isotropic emission component at 137 $\mu \mathrm{m}$. At $262 \mu \mathrm{m}$, separation to individual emission components is not easy because of a rather low signal to noise ratio, and only the upper limit was obtained for the isotropic emission.

Figure 6 shows the residual isotropic emission obtained by the Nagoya-Berkeley experiment, in which the data of the three long wavelength channels are also included. It should be mentioned that the isotropic emission in Figure 6 is for the lowest case. For example, it cannot be excluded that the main part of the residual emission at $102 \mu \mathrm{m}$ is this unknown component. In this case the isotropic emission thus obtained is consistent with the IRAS isotropic emission, but the ZL-thermal at the anti-solar region must be much darker than that expected from the model.

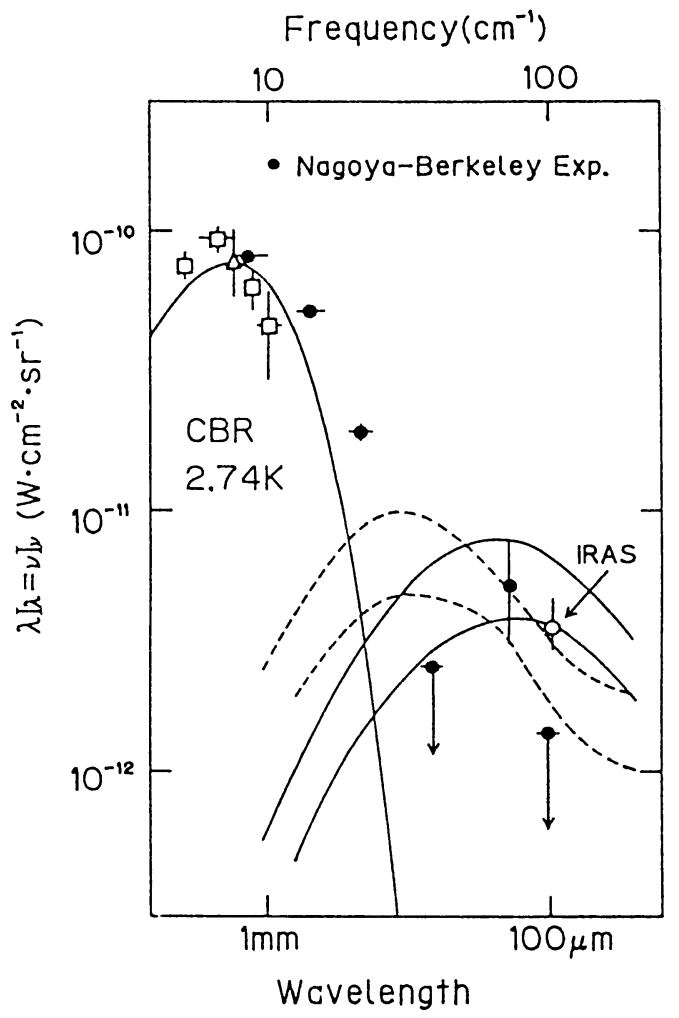

Figure 6. Isotropic emission observed by the Nagoya-Berkeley experiment (filled circles). IRAS background by Boulanger and Perault (1988) is shown by an open circle. Models which include a large evolution effect for galaxies are indicated by the solid and dashed lines (Rowan-Robinson and Carr, 1988).

There are two important features in Figure 6. One is the very low brightness at $262 \mu \mathrm{m}$, which provides a serious constraint on models used to interpret the submillimeter excess. For example, a Comptonization model with relativistic electrons is excluded by this data point (Wright, 1981; Hayakawa et al., 1987). Another is that the isotropic emission at 100-300 $\mu \mathrm{m}$ has a fairly narrow spectral width. Rowan-Robinson and Carr (1988) proposed a model to 
explain the IRAS background by the integrated light from distant galaxies, including a large evolution effect. The solid lines and dashed lines in Figure 6 represent the contribution of the star burst galaxies and cirrus emission of the normal galaxies, respectively. As is seen in Figure 6 , the integrated light of the galaxies with continuous redshift distribution inevitably results in a very broad spectral width. If the $100-300 \mu \mathrm{m}$ background is cosmological, a very narrow spectrum feature at a fixed redshift is required.

The far-infrared background may be of galactic origin. One possibility is to assume a dust component which is distributed uniformly in the Galaxy and has no correlation with neutral hydrogen. In order to search for the EBL, more detailed observations of the spectrum and its spatial variation are necessary. In particular, observations of the sky around $300 \mu \mathrm{m}$ seem very important, since the sky is very dark and the galactic emission has small contribution.

In the submillimeter region close to $1 \mathrm{~mm}$, excess emission over $2.74^{\circ} \mathrm{K}$ blackbody spectrum (Matsumoto et al., 1988b) was found. This submillimeter excess requires $10 \%$ more energy density for the cosmic background, which suggests a vast energy release in the early universe. The details of the model and theory are well described by De Zotti in these proceedings.

\section{FUTURE PROSPECTS}

Several space missions are now in progress to measure the infrared background. COBE (Cosmic Background Explorer) is the biggest one among them and will definitely provide valuable data on the infrared EBL. FIRAS will delineate the submillimeter spectrum, while DIRBE will be very useful to observe the near-infrared and far-infrared background. Measurement of the foreground emission by COBE will also be important for future missions.

The Nagoya group is now preparing for two rocket flights. One is a submillimeter experiment in collaboration with U. C. Berkeley, which will be launched in August 1989. Another is focused to measure the spectrum of the near-infrared background and will be launched in February 1990.

Japan is developing a small cooled infrared telescope (IRTS) on board the small space platform (SFU: Space Flyer Unit) which will be launched in 1994. Four focal plane instruments are being prepared in collaboration with the U.S.A. IRTS is dedicated to observing the diffuse extended emission from the near-infrared to the submillimeter range. IRTS has a great capability for the infrared cosmic background and will play a role in the advanced mission of COBE. Details of IRTS are given by Okuda in this volume.

The infrared cosmic background is one of the key observations in cosmology, although observational evidence is not enough at present. In the coming decade, the missions described above will provide the crucial views for understanding the structure and evolution of the universe.

\section{REFERENCES}

Akiba, M., et al. 1989, submitted to Publ. Astr. Soc. Japan.

Boughn, S. P., et al. 1986, Ap. J., 301, 17.

Boughn, S. P., and Kuhn, J. R. 1986, Ap. J., 309, 33.

Boulanger, F., and Perault, M. 1988, Ap. J., 330, 964.

Dube, R. R., et al. 1977, Ap. J. (Letters), 215, L51.

Harwit, M., et al. 1966, A. J., 71, 1026.

Hauser, M. G., et al. 1984, Ap. J. (Letters), 278, L15.

Hayakawa, S., et al. 1970, Space Res., 10, 248. 
Hayakawa, S., et al. 1987, Pub. Astr. Soc. Japan, 39, 941.

Hofmann, W., and Lemke, D. 1978, Astr. Ap., 68, 389.

Hofmann, W. et al. 1973, Nature Phys. Sci., 243, 140.

Lange, A., et al. 1989, submitted to Ap. $J$.

Matsumoto, T., et al. 1988a, Ap. J., 329, 567.

Matsumoto, T., et al. 1988b, Ap. J., 332, 575.

Partridge, R. B., and Peebles, P. J. E. 1967, Ap. J., 148, 377.

Rowan-Robinson, M., and Carr, B. 1988, Post-Recombination Universe, ed. N. Kaiser and A. N. Lasenby, 125.

Terebey, S., and Fich, M. 1986, Ap. J. (Letters), 309, L73.

Toller, G. N. 1983, Ap. J. (Letters), 266, L79.

Yoshii, Y., and Takahara, F. 1988, Ap. J., 326, 1.

Wright, E. L. 1981, 250, 1.

Ch. Leinert: Is it conceivable that part of the isotropic near-infrared emission you measured is due (a) to zodiacal light (b) to instrumental scattered light?

T. Matsumoto: (a) It is not impossible to attribute the isotropic emission to zodiacal light. But in this case, zodiacal light should have a narrow peak around the K-band. Previous observations on the cometary dust show no indication of this. (b) The off-axis response of the instrument was measured down to a level of $10^{-10}$ in the laboratory. The galactic north pole region was $100^{\circ}$ away from the earth's limb; therefore instrumental scattering is negligible.

K. Mattila: (1) Is the Galaxy model at $2.4 \mu \mathrm{m}$ really known accurately enough to allow a cosec bmodelling and separation of the extragalactic background light? (2) Concerning the sub-millimeter results, I have an uncertain feeling for the method of plotting your surface brightness vs. $N_{H I}$. As we have heard this morning there are dramatic anti-coincidences between dust and HI. Thus the method of extrapolating to $N_{H I}=0$ may be questioned.

T. Matsumoto: (1) We constructed our model of the Galaxy by using the luminosity function and spectral type distribution. This model was checked by our own data. The observed brightness at the Jband was consistent with being the sum of zodiacal light and star light. At the K-band, however, we can't explain the observed brightness with this model and needed an additional isotropic emission. In other words, if we attribute this isotropic emission to stars, the color of the starlight had to be extremely red, which is quite different from what is observed in external galaxies. (2) Yes. But I proposed the possibility that the dust is distributed uniformly at high galactic latitudes. 